\title{
The need for long-term empirical studies in remote interpreting research: a case study of telephone interpreting
}

\author{
Leong Ko
}

The University of Queensland

Remote interpreting refers to the mode of interpreting in which the interpreter and clients do not meet in person and interpreting is conducted via media such as telephone or the Internet. Remote interpreting is becoming increasingly popular globally, particularly for community interpreting. However, this area is significantly under-researched and some of the findings of the limited existing research and from subjective observation do not accurately reflect the real situation. The findings of a long-term empirical study of sound-only telephone interpreting suggest that telephone interpreters can overcome the problems of fatigue, stress, and a reduced concentration span after a period of interpreting practice and by using proper equipment. The paper concludes that long-term empirical studies are an essential methodology in remote interpreting research.

\section{Introduction}

Remote interpreting refers to the mode of interpreting in which the interpreter and the people being interpreted for (i.e. the clients) do not meet face-to-face and interpreting is conducted via media such as the telephone, the Internet, videoconferencing and other forms of telecommunications technology that can enable interpreting to take place. Remote interpreting is becoming increasingly popular on the global interpreting market, particularly for community interpreting in the consecutive mode. For instance, it is widely used in community interpreting settings such as police interviews, medical consultations, immigration interviews, and for many kinds of enquiries. Remote interpreting has many advantages, including convenience for users of the service, access to interpreters in minor languages or specialized areas who are not available for face-to-face interpreting, removal of distance even across countries, and presumably increased cost effectiveness for both service users and interpreters.

However, remote interpreting is significantly under-researched and most of the limited research that has been done is not based on long-term empirical studies. There have been a number of findings from individual investigations, short-term experiments and personal observations about the features and constraints of remote interpreting. Some of these findings are subjective and are yet to be tested by long-term empirical studies to determine whether they reflect the true features of remote interpreting and are applicable to actual remote interpreting practice. This research paper concentrates on sound-only telephone interpreting and is based on a long-term 
empirical study, involving a group of six interpreters carrying out simulated telephone interpreting in eight sessions over a total of 24 hours. The paper also incorporates an analysis of a survey of five practising telephone interpreters. Issues examined in the study include the fatigue and stress associated with telephone interpreting, such as the reported shorter concentration span of interpreters, the telephone equipment, and the lack of visual interaction.

\section{An overview of remote interpreting, with an emphasis on telephone interpreting}

With the development of telecommunications technologies, the use of remote interpreting has grown rapidly in recent years. In terms of the technologies involved, remote interpreting can generally be classified into three categories: interpreting via the Internet, interpreting via local area networks (LANs) - i.e. internal or designated networks for audioconferencing or videoconferencing - and interpreting via the telephone. This paper will not explore in any detail technological issues such as bandwidth, although some general features of interpreting using these technologies will be discussed. For a detailed discussion of these technologies, please refer to Ko (2006: 67-96).

In the case of interpreting via the Internet, the interpreter and the people who require the interpreting service - i.e. the clients - use the Internet connection to provide and receive the interpreting service. Interpreting via the Internet can be sound only, or sound and vision. In the former case, the interpreter and the clients can communicate only verbally with one another, and have no visual interaction. In the latter case, the interpreter and the clients will be able to see one another in addition to their verbal communication. There are a number of companies around the world that provide interpreting services via the Internet, such as SignOn LLC (2006) and Berlitz Interpretation Services (2001).

The second type of remote interpreting is conducted via an internal or designated network. Such a network is often set up or requested by large organizations such as multinational companies or universities, for internal communications such as holding board meetings for company directors who are at different sites or conducting lectures for students at different campuses or centres. The internal or designated network prevents access of outside users. Usually, there are studios or meeting rooms with the necessary equipment and technical backup such as cameras, projectors, large screens and voice points. The voice point is a free-standing telephone device that is placed on a table so that people can listen and talk without using headphones or handsets. By using this technology, the interpreter and clients can see and talk to one another. The speed of transmission is usually faster, as they are likely to be the only group of people using the network at any given time. 
Telephone interpreting is the third form of remote interpreting. It can be divided into sound-only interpreting and picture-phone interpreting. In sound-only interpreting, the interpreter and clients use the conventional telephone to communicate. The communication can be in three or more directions (i.e. with three or more participants) with the interpreter and clients in different places, depending on the bridge capacity of the telephone system (cf. Ko 2006). The picture-phone has the added dimension of showing images of the speakers. It is mainly used for interpreting for the hearing impaired. In this paper, telephone interpreting refers to sound-only interpreting.

Telephone interpreting requires only minimal technological facilities and technical support. For instance, for an interpreted conversation between two clients, the only requirements are telephone lines, telephone sets and a three-way telephone conversation function, which is now easily and widely available by arrangement with telephone companies. Depending on the requirements of the clients, there are multiple-way conversation facilities that can accommodate more than three participants. Telephone interpreting is therefore well suited to community interpreting. It can be used extensively in various community interpreting settings, such as a medical consultation between a doctor and a patient or an interview between an immigration officer and a migration applicant. As a result, the use of telephone interpreting is growing rapidly in countries such as Australia, the United States, Great Britain and many European countries (Pointon, Ozolins \& Doucouliagos 1998; Niska 1999). According to statistics, telephone interpreting services provided by all known operations in the world increased from 102,000 minutes per annum in 1973 to $21,833,000$ minutes per annum in 1995 and were expected to increase to 71,432,000 minutes per annum by 2001 (Pointon, Ozolins \& Doucouliagos 1998: 181). More recent statistics are not available, but the figure is believed to have increased since 1998. Telephone interpreting is provided by a large number of interpreting service providers such as Network Omni Multilingual Communications (2006), Victoria Interpreting and Translation Services (2006) in Australia, On-call Interpreters and Translators Agency (2006) in Australia, Department of Immigration and Multicultural and Indigenous Affairs of Australia (2006), Avalon Language Services (2006) in the US, HSE Language Services (2006) in the UK, Telephone Interpreting Service of South Africa (2006), Language Line Services of AT\&T (2006) in the US and Lionbridge (2006) in the US. The demand for telephone interpreting is growing rapidly.

The reason for this strong demand is that this particular form of interpreting has many advantages. Firstly, it is cost effective and saves time. Interpreters and their clients do not have to travel to a particular site, and so the cost and time spent on travelling is saved. Even the distance between countries can be removed by using telephone interpreting. The telephone "now reaches nearly $100 \%$ of the population in the developed economies" (Pointon , Ozolins \& Doucouliagos 1998: 18). Telephone calls, including interstate calls, are relatively cheap. Even international calls between many 
countries are now very affordable, and in some cases they are even cheaper than interstate calls, including calls from Australia to Hong Kong, China, the US, Canada, New Zealand, Singapore and Malaysia using telephone cards. This means that telephone interpreting is cheaper than on-site interpreting. Secondly, the use of telephone interpreting makes it easier to access interpreters in minor languages (Fors 1999) or interpreters with special expertise, for instance in a particular technical field. Thirdly, it resolves problems in some delicate situations in which the use of an interpreter of the opposite sex is preferred, such as in medical examinations, or the presence of an interpreter is considered inappropriate legally or culturally, for instance, interpreting for a murder case in a small community where people may know one another. Indeed, interpreting by telephone is a "most effective way of getting language services in situations where it might have been impossible to provide an interpreter in person..." (Ozolins 1991: 24). Finally, the telephone interpreting service is well accepted by the clients with a high satisfaction rate. For instance, according to Pointon, Ozolins \& Doucouliagos (1998: 75), the client satisfaction rate (from good, very good to excellent) exceeded $95 \%$ of client organizations surveyed. According to another report on telephone interpreting by $\mathrm{Heh}$ and $\mathrm{Hu}$ (1998: 52-62) in AT\&T Language Line Services in the United States, the telephone interpreting service has generated a high satisfaction rate not only among the clients, but also among the interpreters, as the report points out that statistically customer satisfaction increased from between $87-89 \%$ in 1994 , to $89-91 \%$ in 1995 and $91-93 \%$ in 1996, and the ratings of job satisfaction from the perspective of interpreters were 85,79 and 76 in the respective years, exceeding that of U.S. High Performance Norms.

\section{Findings about remote interpreting, including telephone interpreting}

Although remote interpreting including telephone interpreting has many advantages, past research has found it to face some constraints. The two most common constraints that have been reported are the fatigue or stress experienced by interpreters, and their short concentration span when performing remote interpreting both with and without visual interaction. For instance, both Kurz (1999) and Fors (1999) conclude in their reports that remote interpreting is much more fatiguing and stressful than face-to-face interpreting. Kurz (1999: 116) further points out that remote interpreting "should preferably be confined to short periods", while Fors (1999: 117) concludes that " $[\mathrm{r}]$ emote interpreting puts higher demands on the interpreters, and because of that, only the best interpreters should be allowed to work remotely". It is important to note that Kurz's findings were based on two short experiments in which the subjects were asked to provide a simultaneous interpretation of a lecture for 20 minutes using videoconferencing and 10 minutes in a sound-only situation. This is much like providing an opportunity for interpreters to have hands-on experience with remote interpreting. Although this research has generated some initial findings, it has yet to 
prove that the duration of the experiment is long enough to validate its outcome. For example, after a longer period of remote interpreting, interpreters may become less tired and stressed as they become used to this different mode of interpreting. Fors's findings were based on the observations of the service provider, service user and the interpreter, but Fors did not stipulate how long the interpreters had been engaged in interpreting. Mouzourakis has also identified stress as a major problem in remote interpreting, and has concluded that remote interpreting "will always remain more tiring and stressful" (Mouzourakis 1996: 37).

However, in a study on live simultaneous interpreting on TV, Mack makes an interesting comment that "[i]n most cases the interpreter goes on the air only for a few minutes, but there are also cases with virtually nonstop interpreting for hours", although Mack also emphasizes that it is stressful to interpret on TV for speakers who are thousands of miles away from the TV studios (Mack 2001: 129-130). This seems to suggest that although remote interpreting on TV is stressful, interpreters can manage to interpret for hours. It is important to note that the interpreters in Mack's report are physically present on TV while doing simultaneous interpreting remotely. Indeed, this can be more stressful than interpreting from TV screens. Mack's research is based on interviews with a small group of simultaneous interpreters who regularly work for TV stations. However, in Mack's paper, the claim that interpreters can interpret for hours was mentioned in passing without further elaboration. It therefore remains uncertain how the interpreters coped with hours of remote interpreting and what difficulties they experienced.

Similar concerns about fatigue in sound-only telephone interpreting have been expressed by two major organizations in Australia that provide telephone interpreting services in community settings - Victoria Interpreting and Translation Services of Australia and On-call Interpreters and Translators Agency of Australia. In 2002, the author interviewed the managers of these two interpreting service providers, who expressed the view that the duration of telephone interpreting should not exceed 20 minutes because interpreters would experience fatigue in this working environment. However, this claim was based on their experience as managers of telephone interpreting service providers rather than as telephone interpreters who actually work in the field. Indeed, if the issue of the remote interpreter's fatigue or stress levels and shortened concentration span (in comparison with other forms of interpreting) is assessed based only on whether interpreters can have visual interaction, it could be argued that the interpreters would feel more tired and stressed and have an even shorter concentration in sound-only telephone interpreting. For instance, as Kurz (1999: 116) reports, "the sound-only situation (...) was considered to cause even greater fatigue", although Kurz's experiment focused on simultaneous interpreting.

It is important to note that all of these findings have been collected not from long-term empirical studies, but from short experiments or obser- 
vations. Therefore, their validity is doubtful. In a paper about remote interpreting coordinated by Niska (1999), Gile made an important point about the nature of such research or observations:

Measurement of subjective feelings of fatigue and stress is clearly too unreliable in this respect, but even 'objective' measurements of performance may suffer. Is it not possible that interpreters who fear remote interpreting will unconsciously perform less well in this mode? Is it not possible that interpreters who are happy about the new technology and its prospects will perform better? (...) Taking full advantage of the tools and environment of remote interpreting may take some time to learn. (Gile 1999: 120)

Gile further suggested that empirical research was necessary in this regard (ibid.). Mouzourakis (1996) also admits that more research is needed, despite making several conclusive remarks about the constraints of remote interpreting. Macleod (2000), who used the telephone for tutoring purposes in distance learning, emphasizes that stress is often found in initial experiences with telephone conferences. Therefore, it can be hypothesized that remote interpreters may be able to overcome the problems of fatigue and stress and achieve a longer concentration span after a sufficient period of interpreting practice or special training in this particular working environment.

\section{A long-term empirical study of telephone interpreting}

In 2002, I conducted a research project on teaching interpreting by distance mode (cf. Ko 2004). As part of the project, I conducted an empirical study of telephone interpreting by asking six interpreters to carry out simulated telephone interpreting in eight sessions over four weeks for a total of 24 hours. Each interpreting session lasted for three hours with breaks in the middle depending on the requirements of participants. The interpreters were randomly selected with no specific selection criteria except that they did not have any experience in telephone interpreting. One of the major modes of interpreting used in this study was dialogue interpreting, which is also one of the most common forms of community interpreting. For the dialogue interpreting practice, the participants broke into two groups, with three participants in each group. One participant acted as an English speaker, another as a non-English speaker and the third as the interpreter. I was involved as an observer and sometimes as a speaker but not as an interpreter. The participants were required to use a telephone with a 3-way conversation facility. I had access to a telephone system with a capacity to accommodate eight telephone lines (including mine) at the same time. The data were collected from the participants' diary entries, which were made at the end of each interpreting session, questionnaires and interviews at the end of the study, and 
my observation records. In the diary, the participants were asked to answer some specific questions and to make their own comments.

This empirical study generated a number of interesting findings. The most important finding relates to the interpreters' concentration span, which is directly relevant to the issue of the fatigue reportedly experienced by telephone interpreters. The question and responses in the diary regarding this issue are as follows:

"I could concentrate comfortably on interpreting practice continuously for: $15 \operatorname{mins}$ [ ], 30 mins [ ], 45 mins [ ],60 mins [ ]”

Table 1 - Interpreters' concentration spans

\begin{tabular}{|l|c|c|c|c|}
\hline & \multicolumn{5}{|c|}{ No of interpreters } \\
\hline & 15 minutes & 30 minutes & 45 minutes & 60 minutes \\
\hline Session 1 & 5 & 1 & & \\
\hline Session 2 & 3 & 3 & & \\
\hline Session 3 & 2 & 4 & & \\
\hline Session 4 & 1 & 3 & 2 & 1 \\
\hline Session 5 & 1 & 1 & 3 & 1 \\
\hline Session 6 & & 2 & 3 & 2 \\
\hline Session 7 & & 1 & 3 & 3 \\
\hline Session 8 & & 1 & 2 & \\
\hline
\end{tabular}

The results show that in the first session, almost all of the interpreters felt tired after interpreting for 15 minutes, and only one (16.7\%) could manage to interpret for up to 30 minutes. However, in the second and third sessions, the situation began to improve marginally. This indicates that although the concentration spans of all of the interpreters still ranged from 15 to $30 \mathrm{~min}-$ utes, the number of interpreters who were beginning to adapt to the new interpreting mode was showing a slow but steady increase. With more practice, the concentration spans of all of the interpreters increased. In the seventh session - i.e. after 18 hours of practice - all but one interpreter were able to interpret with comfort via the telephone for 45 to 60 minutes.

These findings suggest that, after a period of interpreting practice, the interpreters were able to overcome the problem of fatigue associated with working over the telephone and increase their concentration span to as long as 45 to 60 minutes. However, in the beginning, especially in the first three sessions or 9 hours, the interpreters did experience difficulties and feel tired. This implies that the experiments conducted by Kurz coincided exactly with the stage at which interpreters had the lowest level of performance. It is therefore natural that the interpreters felt tired, stressed and frustrated. Interpreters simply need practice to become accustomed to telephone interpreting. These findings also contradict the claims made by the two telephone interpreting service providers in Australia about the optimum length of telephone interpreting. Furthermore, they suggest that it is not necessary to use the most competent interpreters for remote interpreting, as claimed by Fors. The interpreters involved in my study were not screened for inter- 
preting competence. Rather, they were 'ordinary' interpreters. After practice, the great majority of them were able to achieve a concentration span of 45 minutes or more in telephone interpreting.

Another important finding relates to the equipment used in telephone interpreting. Currently, there are many kinds of telephone equipment that can be used for telephone interpreting, including handset telephones, cordless phones, mobile phones, telephones with headphones, speaker phones and voice points. The last two are free-standing forms of telephone equipment that are placed on a table to enable participants to listen and talk without the use of headphones or handsets. However, since interpreters normally take notes while interpreting, telephone equipment can be classified according to whether or not it allows interpreters to use their hands freely instead of holding the telephone or receiver. There are therefore basically two categories of telephone equipment: (1) those that allow users to move their hands freely, such as handset telephones or mobile phones with headphones, speaker phones and voice points; (2) those that require users to hold the telephone or receiver such as handset telephones, cordless phones or mobile phones without headphones.

It was found in the experiment that different types of equipment vary significantly in terms of their impact on the interpreter's comfort level in interpreting. During the initial three interpreting sessions, four interpreters used conventional handset telephones or cordless phones without headphones, one used a handset telephone with headphones and one used a speakerphone. Those who used the telephone equipment without headphones reported that it was difficult and clumsy to interpret because they had to hold the receiver while they were taking notes. In fact, all those who had a shorter concentration span in the initial three sessions did not have headphones or speakerphones. From the fourth session on, all of the interpreters had headphones or speakerphones, and the problem of discomfort with telephone equipment was not reported again.

One minor issue identified in the experiment was that there was signal interference when using the cordless phone - namely, there was a rattling or piercing noise in the background. This affected not only the particular interpreter who used the cordless phone but the whole group. When the interpreter stopped using the cordless phone, the problem disappeared. However, this problem relates to the quality of signal transmission and is therefore technical. If the quality of transmission improves, the problem should be solved.

Lack of visual interaction is an important issue in remote interpreting, particularly in sound-only telephone interpreting. In face-to-face interpreting situations, visual interaction helps interpreters obtain accurate information, determine the effectiveness of the communication, and facilitate its progress. For instance, it helps interpreters make correct judgments about the interpreting situation and decide whether they should intervene (e.g. Gentile, Ozolins \& Vasilakakos 1996; Wadensjö 1992; Roy 2000). However, in sound-only telephone interpreting, interpreters do not have this access and need to rely solely on verbal interaction for all communication purpos- 
es. The lack of face-to-face contact in remote interpreting has been raised as a concern by several researchers, including Kurz (1999), Fors (1999), Wadensjö (1999) and Mack (2001). Wadensjö (1999: 254) pointed out that interpreters were not able to capture "the communicative cues provided by interlocutors' gestures, posture, mimics, and other non-verbal behaviour, all of which have a role in guiding the interpretation". It is likely that telephone interpreting was considered more tiring and stressful than face-to-face interpreting because of this lack of visual interaction, because this is the major difference between the two forms of interpreting.

In the diaries used in this research, the interpreters were asked to respond to a particular statement relating to this issue: "Lack of visual interaction did not affect my interpreting practice." The following table shows their responses:

Table 2 - The impact of lack of visual interaction

\begin{tabular}{|l|c|c|c|c|c|}
\hline & $\begin{array}{c}\text { Strongly } \\
\text { agree }\end{array}$ & Agree & $\begin{array}{c}\text { Neither agree } \\
\text { nor disagree }\end{array}$ & Disagree & $\begin{array}{c}\text { Strongly dis- } \\
\text { agree }\end{array}$ \\
\hline Session 1 & 1 & 3 & 2 & & \\
\hline Session 2 & 1 & 3 & 2 & & \\
\hline Session 3 & 3 & 2 & 1 & & \\
\hline Session 4 & 3 & 1 & 2 & & \\
\hline Session 5 & 4 & 1 & 1 & & \\
\hline Session 6 & 3 & 2 & 1 & & \\
\hline Session 7 & 4 & 1 & 1 & & \\
\hline Session 8 & 4 & 1 & 1 & & \\
\hline
\end{tabular}

The data show that in the initial two sessions, only one interpreter $(16.7 \%)$ felt at home interpreting in the new environment and experienced no difficulties, three interpreters $(50 \%)$ indicated that they "agreed" rather than "strongly agreed" with the statement, which suggests that they generally did not feel a strong impact from the lack of visual interaction but were not totally comfortable with the environment, and two interpreters $(33.3 \%)$ were uncertain, but did not feel a serious negative impact. The situation improved gradually from the third session. However, it was not until the fifth session that the great majority of interpreters (i.e. those who ticked "strongly agree" and "agree") became accustomed to interpreting without visual interaction. As discussed earlier, it was also at around this stage that all of the interpreters were using headphones or speakerphones. This means that the improvement they reported also related to the telephone equipment. However, one point of note is that even after eight sessions, there was still one interpreter $(16.7 \%)$ who was not completely confident with interpreting without visual interaction. This indicates that there may be a small percentage of interpreters who may not be fully suited to telephone interpreting or who may require more time to get used to this form of interpreting.

The data also closely match the findings on improvements in concentration span, and therefore confirm that while it is true that the lack of visual interaction had some effect on the interpreters in the study, this was mostly the case in the initial stages, when the interpreters were not familiar with 
this mode of interpreting and did not have the appropriate equipment. This constraint can be overcome after a period of interpreting practice or training and by using proper telephone equipment.

It is important to note that this lack of visual interaction is inherent in the nature of sound-only telecommunications equipment. In other words, if sound-only telephone equipment is used, communicative cues of a non-verbal nature cannot be conveyed or received. This is an issue not only for the interpreter, but also for the clients. It is a problem that both parties need to overcome. The author observed that the interpreters participating in this study were aware that visual interaction would not be possible in the experiment, and were therefore prepared to treat telephone interpreting as a new mode of interpreting and accept the challenges involved. This positive approach enabled them to find ways to adapt to this new form of interpreting rather than continually comparing it with face-to-face interpreting, searching for and relying on visual interaction.

In fact, even if telecommunications equipment capable of accommodating some level of face-to-face contact, such as videoconferencing, is used in remote interpreting, the transfer of communicative cues of a nonverbal nature will be limited and will differ from interpreting in an actual face-to-face situation. For instance, if a TV screen or Web camera is used in interpreting via videoconferencing or the Internet, the transfer of non-verbal communicative cues will be limited to what a TV screen or computer monitor can display - perhaps little more than the interlocutors' facial expressions. However, non-verbal communicative cues include gestures, posture, mimics and other non-verbal behavior (Wadensjö 1999) rather than just facial expressions. In addition, there are some situations in which a number of clients are present in one place. It would be "very hard to obtain acceptable quality with more than 6-7 persons in each studio" (Mouzourakis 1996: 27). So unless a very large screen is used, non-verbal communicative cues cannot be adequately displayed and captured, and the visual interaction will not really be satisfactory. Interpreters in this situation may choose to ignore such confusing and even distracting non-verbal communicative cues and concentrate on verbal communication.

\section{Survey of practising telephone interpreters}

In the course of my research, I conducted a survey of five practising interpreters in Australia who work both as on-site and telephone (sound-only) interpreters. I asked them the following three questions, and also invited them to make their own comments:

- What kind of telephone equipment do you use in telephone interpreting?

- How long can you interpret comfortably over the phone?

- What is the longest telephone interpreting time you have experienced and how did you feel? 
It was found that these interpreters used whatever telephone equipment that was at their disposal when they received calls for telephone interpreting, except when a telephone interpreting session was pre-booked, in which case they could decide on the most appropriate telephone equipment for the job. The telephone equipment they used included handset telephones, cordless phones and mobile phones with or without headphones, speakerphones, and voice point when it was available - e.g. provided by the organization that required telephone interpreting in its office.

All of the interpreters agreed that they could interpret comfortably for around 30 minutes using any kind of telephone equipment without experiencing fatigue. However, if a job lasted for more than one hour, they observed that they definitely preferred to use a speakerphone or voice point.

With regard to their longest telephone interpreting session, one interpreter indicated one hour, two interpreters indicated 1.5 hours, and the other two indicated an hour and fifty minutes and two hours respectively. All of them, including the interpreter whose longest telephone interpreting experience was one hour, felt that they could cope with one hour of telephone interpreting with ease, but that they would prefer an interpreting job not to exceed 1.5 hours.

My own experience with telephone interpreting confirms the above claims, especially in relation to a preference for using the voice point for longer interpreting jobs. I come from an area where people speak a dialect that is not commonly understood by people from other parts of the country. On a number of occasions, the Refugee Review Tribunal in Australia has required an interpreter to interpret at hearings for applicants from this area. Due to the fact that I am the only professional interpreter who can speak this dialect in Australia and that the Member of the Tribunal, the applicant and I are often in three different cities, telephone interpreting is the only way to facilitate such hearings. The hearings usually last for two to three hours. For two-hour sessions, there is usually no break, but if a hearing lasts for three hours, a break of 10 minutes or so is often arranged. During the hearing process, I interpret alone in a room using the voice point. I have found it very comfortable to interpret using the voice point. Since I am the only person in the room and do not have visual contact with anyone, I do not have to worry about my personal image or maintain eye contact with anyone. The voice point allows me to take notes without any constraints, move around and stretch when I need to, and press the "mute" button if I need to cough or take a drink. I find this much less stressful than face-toface interpreting in a similar situation, where the Member and his or her secretary sit in front of me and the applicant and his or her lawyer are next to me. Apart from the first time, when it took me around 10 minutes to get used to this type of sound-only communication and the functions of the equipment, I have not experienced any difficulty interpreting for two to three hours in this technical environment. In saying this, I do not mean to imply that telephone interpreting is easier than face-to-face interpreting, 
only that it is different. To me, lack of visual interaction is both an advantage and a disadvantage. The disadvantage is that I cannot see my clients and do not have visual interaction, while the advantage is that I can relax and do not have to maintain eye contact with them.

Admittedly, this survey is limited because of the small number of interpreters involved, and my personal experience can only be regarded as an individual case. However, the results of the survey and my anecdotal experience lend some support to the findings of the empirical study that:

- the lack of visual interaction can be overcome by telephone interpreters;

- the problem of fatigue can also be overcome, especially by using proper telephone equipment;

- $\quad$ and that the interpreter's concentration span is longer than the 10 or 20 minutes that has been claimed in other research and observations.

\section{Conclusion}

The findings of the empirical study and the author's survey of practicing telephone interpreters confirm Gile's observations about the unreliability of subjective feelings about remote interpreting, as well as his view about the time required to become accustomed to and take full advantage of the particular characteristics of remote interpreting. The findings further suggest that satisfactory performance by telephone interpreters is related to use of the appropriate equipment and relevant practice or training. Based on these findings, it can be concluded that telephone interpreters can achieve the concentration span required for telephone interpreting without feeling tired and stressed after undergoing a period of interpreting practice of around 18 hours, and by using proper telephone equipment. An interpreter's concentration span when performing telephone interpreting can be 60 minutes or longer. It can also be concluded that long-term empirical studies are an essential methodology in remote interpreting research.

The findings of other researchers' studies and the observations of interpreting service managers, together with the results of the empirical study and the opinions expressed by practising telephone interpreters, also convey the clear message that remote interpreting is a new mode of interpreting, which is not necessarily more difficult or stressful than face-to-face interpreting, but does differ in some respects. With advances in telecommunications technologies, this mode of interpreting is likely to develop further and interpreters who plan to work in this field will need to learn the new skills associated with this mode of interpreting. A short trial period is not sufficient to grasp the relevant skills, and it may be necessary for the interpreters to undertake a tailor-made training programme.

Finally, the results of this study apply only to sound-only telephone interpreting in community settings, which can be less demanding than other modes of interpreting such as simultaneous interpreting. The study was lim- 
ited to two clients in different places and did not test the situation in which there are two or more clients in one place, such as in a meeting. It is important to point out that a different form of remote interpreting may need an empirical study of a different length, because there are different factors to be assessed, such as the mode of interpreting (e.g. consecutive, simultaneous, or sign language); the type of communication required (e.g. sound only, sound and picture, live and synchronous verbal and visual interaction); and the media to be used (e.g. telephone, videoconferencing and the Internet). All of these variables will have an impact on the appropriate length for an empirical study and will need to be assessed in order to generate valid research outcomes.

\section{Bibliography}

Avalon Language Services (2006). Online at http://www.avalonlanguageservices.com (consulted 17.03.2006).

Berlitz Interpretation Services (2001). Online at http://www.berlitzglobalnet.com (consulted 18.06.2001).

Department of Immigration and Multicultural and Indigenous Affairs of Australia (2006). Online at www.immi.gov.au/tis/how.htm (consulted 17.03.2006).

Fors, Jennie (1999). "Perspectives on remote public service interpreting". H. N. Helge (coord.) "Quality issues in remote interpreting". A.A. Lugris \& A.F. Ocampo (eds). Anovar/anosar estudios de tranduccion e interpretacion, vol. I, Servicio de Publicacion da Universidade de Vigo, 117-119.

Gentile, Adolfo, Uldis Ozolins \& Mary Vasilakakos (1996). Liaison Interpreting - a handbook. Melbourne: Melbourne University Press.

Gile, Daniel (1999). "Assessing the pros and cons of remote interpreting: comments and future prospects from a research perspective". Helge Niska Helge (coord.) "Quality issues in remote interpreting". A.A. Lugris \& A.F. Ocampo (eds). Anovar/anosar estudios de tranduccion e interpretacion, vol. I, Servicio de Publicacion da Universidade de Vigo, 119-121.

Heh, Yung-Chung \& Qian Hu (1998). "Over-the-phone interpretation: a new way of communication between speech communities". American Translators Association's $38^{\text {th }}$ Annual Conference Proceedings, 52-62.

HSE Language Services (2006). Online at http://www.hse.gov.uk/languages/tele_trans.htm (consulted 17.03.2006).

Ko, Leong (2004). Teaching interpreting by distance mode. PhD thesis. University of Queensland.

Ko, Leong (2006). "Teaching interpreting by distance mode: Possibilities and constraints". Interpreting 8(1), 67-96.

Kurz, Ingrid (1999). "Remote conference interpreting: Assessing the technology". H. N. Helge (coord.) "Quality issues in remote interpreting". A.A. Lugris \& A.F. Ocampo (eds). Anovar/anosar estudios de tranduccion e interpretacion, vol. I, Servicio de Publicacion da Universidade de Vigo, 114-116.

Language Line Services (2006). Online at http://www.languageline.com/home.php (consulted 17.03.2006).

Lionbridge (2006). Online at http://www.lionbridge.com/ (consulted 17.03.2006).

Mack, Gabriele (2001). "Conference interpreters on the air: Live simultaneous interpreting on Italian television". Yves Gambier \& Henrik Gottlieb (eds). (Multi) Media translation: Concepts, practices, and research. Amsterdam-Philadelphia: John Benjamins, 25-132.

Macleod, Hamish (2000). "Telephone tutorials in distance learning programmes". Online at http://www.gla.au.uk/Incs/main/FOURTEEN.HTM. (consulted 17.11.2001)

Mouzourakis, Panayotis (1996). "Videoconferencing: techniques and challenges". Interpreting 1(1), 21-38.

NetworkOmni Multilingual Communications (2006). Online at http://www.networkomni.com (consulted 17.03.2006).

Niska, Helge (1999). "Quality issues in remote interpreting". A.A. Lugris \& A.F. Ocampo (eds). Anovar/anosar estudios de tranduccion e interpretacion, vol. I, Servicio de Publicacion da Universidade de Vigo, 109-121. 
On-call Interpreters and Translators Agency (2006). Online at http://www.oncallinterpreters.com (consulted 17.03.2006).

Ozolins, Uldis (1991). Interpreting, translating and language policy: Report to the Language and Society Centre. Melbourne: National Languages Institute of Australia.

Pointon, Tom, Uldis Ozolins \& Chris Doucouliagos (1998). TIS in Europe 1976-2001: Determining the origins, size, nature \& outlook of telephone interpreting services in Europe. London/Melbourne: The Pointon Partnership/Deakin University Centre for Research and Development in Interpreting and Translation.

Roy, Cynthia (2000). Interpreting as a Discourse Process. Oxford: Oxford University Press.

SignOn LLC (2006). Online at http://www.signonasl.com/videotext.htm (consulted 17.03.2006).

Telephone Interpreting Service of South Africa (2006). Online at http://www.dacst.gov.za/ (consulted 17.03.2006).

Victoria Interpreting and Translation Services (2006). Online at http://www.vits.com.au (consulted 17.03.2006).

Wadensjö, Cecilia (1992). Interpreting as Interaction. Linköping: Linköping University. 